\title{
OPTIMAL PRECODING FOR A NOVEL RF-MIMO SCHEME IN TRANSMIT CORRELATED RAYLEIGH CHANNELS
}

\author{
J. Vía, V. Elvira, J. Ibáñez and I. Santamaría \\ Department of Communications Engineering \\ University of Cantabria. 39005 Cantabria, Spain \\ email: \{jvia,victorea,jesus,nacho\}@gtas.dicom.unican.es
}

\begin{abstract}
Recently, a novel MIMO transceiver architecture, which avoids the costly conversion to/from baseband through parallel RF chains, has been proposed. Despite its obvious advantages, the limitations of the analog combining architecture makes necessary to develop specific transmission schemes. For instance, in the case of perfect channel state information (CSI) at the receiver, and correlation CSI at the transmitter, the space and time encoders must operate separately (the former works in the RF domain and the latter works in baseband), and at different time scales: the spatial encoder or RF beamformer must remain fixed during the transmission of a probably large number of symbols, whereas the time encoder can work at the symbol rate. In this paper we propose a transmission scheme for this scenario with the goal of minimizing the pairwise error probability (PEP). In particular, with the proposed scheme the symbols are time-precoded with a unitary discrete Fourier transform (DFT) matrix, and then are successively transmitted using a set of RF weights (beamformers). The optimal spatial precoding matrix containing the $\mathrm{RF}$ beamformers is obtained by matching its left eigenspace with the eigenspace of the channel correlation matrix, applying standard power water-filling along these directions, and choosing its right eigenspace as any unitary matrix with unit-norm elements such as the DFT matrix. Numerical examples illustrate the good performance of the proposed scheme.
\end{abstract}

\section{INTRODUCTION}

The last years have witnessed a steady trend to move the baseband of digital communications systems as close as possible to the antenna. However, for multiple-input multiple-output (MIMO) systems, the conversion to/from baseband through parallel RF chains implies that the system cost, size and power consumption is multiplied by the number of transmitting or receiving antennas. In order to mitigate these drawbacks it is interesting to re-think about the pros and cons of moving some processing from the baseband to the radio frequency (RF) front-end.

In previous works we have proposed a novel architecture for ana$\log$ antenna combing in the RF domain, which is shown in Fig. 1. With this topology, after RF signal combining, only one signal path is required for the second mixer/intermediate-frequency chain, the ADCs and the baseband, which obviously translates into a lower system cost and power consumption than that of conventional MIMO

The research leading to these results has received funding from the European Community's Seventh Framework Programme (FP7/2007-2013) under grant agreement $n^{\circ} 213952$ (MIMAX), and by the Spanish Government (MICINN) under projects TEC2007-68020-C04-02 (MultiMIMO) and TEC2007-30929-E.

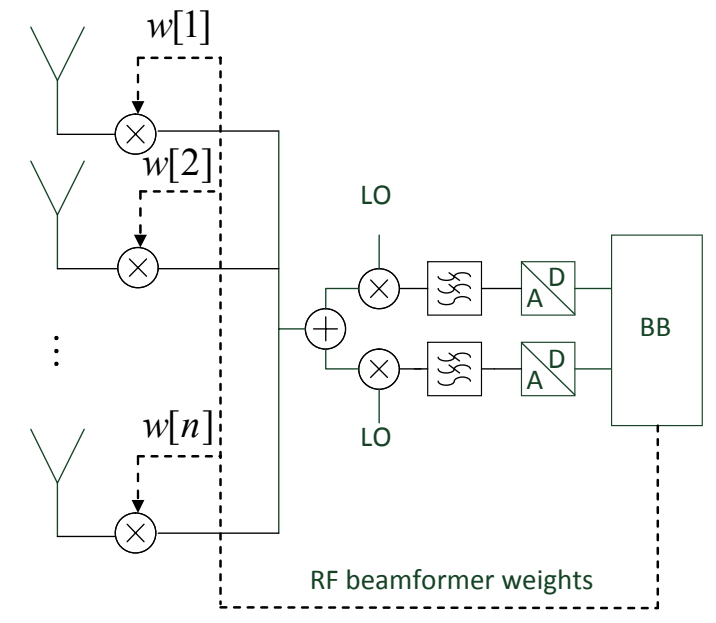

Fig. 1. Analog antenna combining in the RF path for MIMO communications systems. Exemplarily shown for a direct-conversion receiver.

architectures. The development of this type of analog weighting RF circuits in SiGe-BiCMOS technology [1] suitable for mass fabrication is currently being pursued within the EU funded project MIMAX (MIMO Systems for Maximum Reliability and Performance). Specifically, the proposed analog RF topologies allow to achieve precise phase shifters with $360^{\circ}$ control range and an amplitude control with a dynamic margin of at least $20 \mathrm{~dB}$.

From a signal processing point of view, one of the distinguishing features of the novel topology is that the space and time encoders operate separately and at different time scales. Specifically, beamforming (here also referred to as spatial encoder) is performed in the radio frequency domain by the adaptive combiner, whereas time encoding is carried out in the baseband. On the other hand, the complex weights in the RF path change at a much slower rate than the time encoder, which typically works at the symbol rate. Additionally, we must point out that, by performing adaptive combining in the radio frequency domain, the multiplexing gain of the MIMO system is always limited to one. However, it has been recently shown [2-4] that other important benefits of the MIMO channel such as spatial diversity or array gain can be retained by the new architecture.

In this paper, we consider the design of the time and space encoders for minimizing the pairwise error probability (PEP) under perfect channel state information (CSI) at the receiver, and correlation CSI at the transmitter side. Firstly, we show that by using 


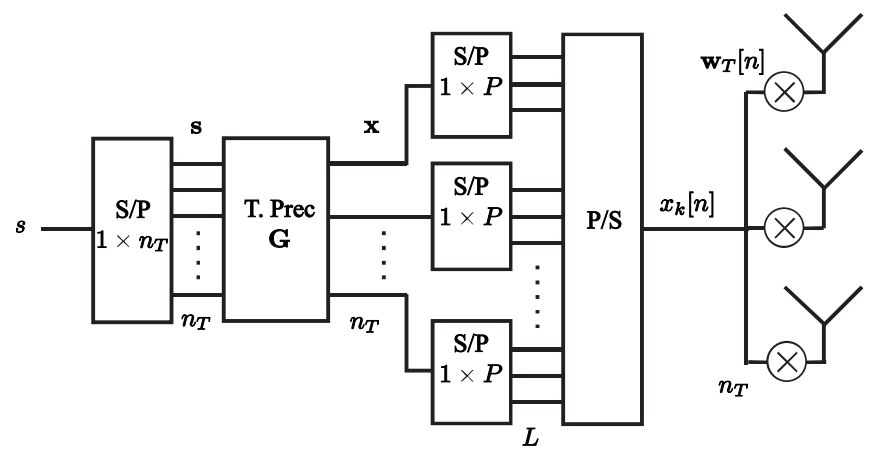

Fig. 2. Transmission scheme. The time-precoded symbols are transmitted using $n_{T}$ different beamformers.

the optimal (maximal ratio combining) receive beamfomers, our RFMIMO scheme can be written as a set of single-input single-output (SISO) channels, which are orthogonal in time. Therefore, our problem reduces to finding separate optimal time and space encoders. Specifically, the optimal full-diversity time encoder consists of a shifted version of the discrete Fourier transform (DFT) matrix. On the other hand, the design of the space encoder resembles the conventional problem of optimal precoding for correlated MIMO channels and, in fact, the solution is rather similar: statistical waterfilling over the strongest modes of the transmit covariance matrix. However, a direct application of this solution to the analog combining architecture would require to change the energy of the RF transmit beamformers through time and, therefore, is not adequate for implementation. To avoid this problem, an additional set of constraints enforcing transmit beamformers of constant energy must be introduced in the optimization problem. These new constraints are satisfied by mixing the strongest modes of the transmit correlation matrix by a unitary matrix with unit-norm elements (the DFT matrix is again used for this purpose). Finally, some simulation examples illustrate the performance of the proposed space-time precoding scheme.

Throughout this paper we will use bold-faced upper case letters to denote matrices, bold-faced lower case letters for column vector, and light-faced lower case letters for scalar quantities. The superscripts $(\cdot)^{T}$ and $(\cdot)^{H}$ denote transpose and Hermitian. $\|\mathbf{A}\|, \operatorname{Tr}(\mathbf{A})$ and $|\mathbf{A}|$ will denote the Frobenius norm, trace and determinant of the matrix A. Finally, $\operatorname{diag}(\mathbf{a})$ denotes the diagonal matrix with vector $\mathbf{a}$ in its diagonal and $\mathbf{I}$ is the identity matrix of the required dimensions.

\section{RF-MIMO SCHEME}

\subsection{Transmission Scheme}

In this paper we consider a flat-fading single-carrier MIMO system with $n_{T}$ transmit and $n_{R}$ receive antennas. The MIMO channel is assumed to be static during the transmission of a frame composed of $L$ symbols, which are transmitted using a set of $n_{T}$ different transmit beamformers, ${ }^{1}$ which are successively applied.

As we already pointed out, due to technological limitations of the RF circuitry, the beamformer weights must remain fixed during the transmission of a probably large number of symbols. The trans-

\footnotetext{
${ }^{1}$ The results in the paper can be easily generalized to any other number of transmit beamformers. However, we choose $n_{T}$ because this is the minimum number of transmit beamformers to achieve full spatial diversity.
}

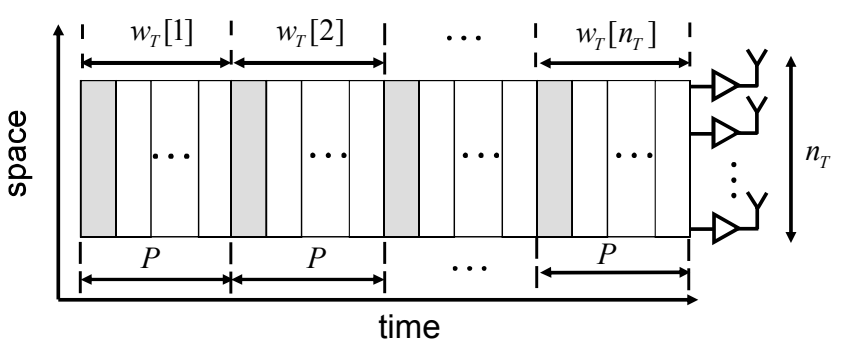

Fig. 3. Frame structure: the grey columns form an $n_{T} \times n_{T}$ codeword that conveys $n_{T}$ time-precoded symbols transmitted through $n_{T}$ different beamformers.

missions scheme and frame structure are depicted in Figs. 2 and 3. Specifically, we assume that a block of $P$ time-precoded symbols are transmitted with each beamformer (i.e., $L=P n_{T}$ ), which yields the following transmission model

$$
\mathbf{c}_{k}[n]=\mathbf{w}_{T}[n] x_{k}[n], \quad k=1, \ldots, P, \quad n=1, \ldots, n_{T},
$$

where $\mathbf{w}_{T}[n]=\left[w_{T, 1}[n], \ldots, w_{T, n_{T}}[n]\right]^{T}$ is the $n$-th transmit beamformer (RF weights) and $x_{k}[n]$ is the $k$-th symbol in the $n$-th block.

Defining now the $n_{T} \times n_{T}$ transmit beamformer matrix (or spatial encoder)

$$
\mathbf{W}_{T}=\left[\mathbf{w}_{T}[1] \cdots \mathbf{w}_{T}\left[n_{T}\right]\right]
$$

and grouping the $k$-th columns of each block within a frame (the grey columns in Fig. 3), we form the following $n_{T} \times n_{T}$ codeword

$$
\mathbf{C}_{k}=\left[\mathbf{c}_{k}[1] \cdots \mathbf{c}_{k}\left[n_{T}\right]\right]=\mathbf{W}_{T} \operatorname{diag}\left(\mathbf{x}_{k}\right),
$$

where

$$
\mathbf{x}_{k}=\left[x_{k}[1], \ldots, x_{k}\left[n_{T}\right]\right]^{T}=\mathbf{G s}_{k}, \quad k=1, \ldots, P,
$$

are the time-precoded symbols, $\mathbf{G} \in \mathbb{C}^{n_{T} \times n_{T}}$ is the time encoding matrix, and $\mathbf{s}_{k}=\left[s_{k}[1], \ldots, s_{k}\left[n_{T}\right]\right]^{T}$ contains the information symbols to be transmitted. Note that with this scheme each codeword $\mathbf{C}_{k}$ can be coded and decoded independently from others.

\subsection{Received Signals}

The signals associated to one codeword at the $n_{R}$ receive antennas $\operatorname{are}^{2}$

$$
\mathbf{Y}=\mathbf{H C}+\mathbf{N}=\mathbf{H W}_{T} \operatorname{diag}(\mathbf{G s})+\mathbf{N},
$$

where $\mathbf{H} \in \mathbb{C}^{n_{R} \times n_{T}}$ is the MIMO channel, which is assumed to be perfectly known at the receiver, and $\mathbf{N} \in \mathbb{C}^{n_{R} \times n_{T}}$ is the complex Gaussian i.i.d. noise.

Although the receiver does not have direct access to $\mathbf{Y}$ due to the analog combining architecture, it is easy to prove that, independently of the time and space encoders used at the transmiter, the optimal receiver is based on the maximal ratio combining (MRC) technique ${ }^{3}$, which applies the receive beamformers

$$
\mathbf{w}_{R}[n]=\frac{\mathbf{H w}_{T}[n]}{\left\|\mathbf{H w}_{T}[n]\right\|} \quad n=1, \cdots, n_{T} .
$$

\footnotetext{
${ }^{2}$ In the rest of the paper and for notational simplicity, we will omit the codeword index $k$.

${ }^{3}$ Notice that MRC beamforming can be applied since we are assuming perfect CSI at the receiver.
} 
Using the MRC receive beamformer, the observations at the output of the RF combiner are given by

$$
\mathbf{y}=\left[\begin{array}{cccc}
\left\|\mathbf{H w}_{T}[1]\right\| & 0 & \cdots & 0 \\
0 & \left\|\mathbf{H w}_{T}[2]\right\| & \ddots & \vdots \\
\vdots & \ddots & \ddots & 0 \\
0 & \cdots & 0 & \left\|\mathbf{H w}_{T}\left[n_{T}\right]\right\|
\end{array}\right] \mathbf{G s}+\mathbf{n},
$$

where $\mathbf{n} \in \mathbb{C}^{n_{T} \times 1}$ is the noise component after MRC beamforming, and $\mathbf{y} \in \mathbb{C}^{n_{T} \times 1}$ is a sufficient statistic for the signals $\mathbf{Y}$ in (1).

\section{DESIGN OF THE TIME AND SPACE ENCODERS}

In this section we describe the main contribution of this paper, which is the design of the time $(\mathbf{G})$ and space $\left(\mathbf{W}_{T}\right)$ encoders that minimize the averaged PEP when correlation channel state information is available at the transmitter. In particular, the channel model is written as

$$
\mathbf{H}=\tilde{\mathbf{H}} \mathbf{R}^{1 / 2},
$$

where $\tilde{\mathbf{H}} \in \mathbb{C}^{n_{R} \times n_{T}}$ has zero-mean unit-variance i.i.d. complex Gaussian elements and $\mathbf{R}$ represents the transmit antenna correlation.

Our optimization problem can be written as

$$
\begin{array}{cl}
\underset{\mathbf{G}, \mathbf{W}_{T}}{\operatorname{Minimize}} & P(\mathbf{s} \rightarrow \hat{\mathbf{s}}), \\
\text { subject to } & \mathbf{G}^{H} \mathbf{G}=\mathbf{I}, \\
& \left\|\mathbf{w}_{T}[n]\right\|=1, \quad n=1, \ldots, n_{T},
\end{array}
$$

where $P(\mathbf{s} \rightarrow \hat{\mathbf{s}})$ denotes the average PEP between two information vectors and the constraints guarantee a constant transmit power.

As will be shown next, an important particularity of the RFMIMO architecture is that the design of the time encoder $\mathbf{G}$ and the spatial encoder $\mathbf{W}_{T}$ are uncoupled problems and can be treated separately.

\subsection{Design of the Time Encoder G}

From the data model in (2), it is easy to see that, for any choice of the transmit beamformers, our communication system reduces to a set of orthogonal SISO channels, which resembles the conventional orthogonal frequency division multiplexing (OFDM) scheme. Thus, in order to achieve full diversity, it is necessary to spread the information symbols $\mathbf{s}$ over the $n_{T}$ time instants and the $n_{T}$ antennas. This spreading is performed by the time encoding matrix $\mathbf{G}$, which can be designed following the ideas discussed in [5-8]. Specifically, assuming maximum likelihood (ML) decoding and QAM constellations, the optimal $\mathbf{G}$ is independent of the specific spatial encoder $\mathbf{W}_{T}$, and it is given by a shifted version of the Fourier matrix

$$
\mathbf{G}=\frac{1}{\sqrt{n_{T}}} \mathbf{F}^{H} \mathbf{D}_{\alpha}
$$

where $\mathbf{F}$ is the $n_{T} \times n_{T}$ Fourier matrix whose $k$-th column is $\mathbf{f}\left(\omega_{k}\right)=$ $\left[1, \exp \left(-j \omega_{k}\right), \cdots, \exp \left(-j \omega_{k}\left(n_{T}-1\right)\right)\right]^{T}$, with $\omega_{k}=2 \pi(k-$ $1) / n_{T}$; and $\mathbf{D}_{\alpha}$ is a diagonal matrix that shifts the frequencies of the Fourier grid an amount $\alpha,{ }^{4}$ i.e.,

$$
\mathbf{D}_{\alpha}=\operatorname{diag}\left(\left[1, e^{-j \alpha}, \ldots, e^{-j\left(n_{T}-1\right) \alpha}\right]\right) .
$$

\footnotetext{
${ }^{4}$ The optimal value of $\alpha$ depends on the codeword size [5, 6].
}

\subsection{Design of the Space Encoder $\mathbf{W}_{T}$}

Taking into account that $\mathbf{y}$ is a sufficient statistic for $\mathbf{Y}$, the analysis of the pairwise error probability can be based on the signals before receive beamforming. Thus, upper-bounding the PEP by means of the Chernoff bound [9] we obtain

$$
P(\mathbf{s} \rightarrow \hat{\mathbf{s}} \mid \mathbf{H}) \leq \exp \left(-\frac{\gamma}{4}\left\|\mathbf{H} \mathbf{W}_{T} \operatorname{diag}(\mathbf{G}(\mathbf{s}-\hat{\mathbf{s}}))\right\|^{2}\right),
$$

where $\gamma$ is the signal to noise ratio. Now, the average of (5) over the channel fading statistics yields [10]

$$
P(\mathbf{s} \rightarrow \hat{\mathbf{s}}) \leq\left|\mathbf{I}+\frac{\gamma}{4} \mathbf{R}^{1 / 2} \mathbf{E}_{\mathbf{s}, \hat{\mathbf{s}}} \mathbf{R}^{1 / 2}\right|^{-n_{R}}
$$

where $\mathbf{E}_{\mathbf{s}, \hat{\mathbf{s}}}$ is the codeword distance product matrix

$$
\mathbf{E}_{\mathbf{s}, \hat{\mathbf{s}}}=\mathbf{W}_{T} \operatorname{diag}(\mathbf{G}(\mathbf{s}-\hat{\mathbf{s}})) \operatorname{diag}(\mathbf{G}(\mathbf{s}-\hat{\mathbf{s}}))^{H} \mathbf{W}_{T}^{H} .
$$

Obviously, the averaged PEP (and therefore the choice of $\mathbf{W}_{T}$ ) depends on the specific pair of information vectors $(\mathbf{s}, \hat{\mathbf{s}})$ considered. However, it seems reasonable $\mathrm{e}^{5}$ to minimize the averaged PEP between the true information vector $\mathbf{s}$ and its closest neighbors $\hat{\mathbf{s}}$, i.e., those vectors which only differ from $\mathbf{s}$ in one element. With this choice, and taking into account that the optimal $\mathbf{G}$ has constant norm entries, the codeword distance product reduces to

$$
\mathbf{E}_{\mathbf{s}, \hat{\mathbf{s}}}=\frac{d^{2}}{n_{T}} \mathbf{W}_{T} \mathbf{W}_{T}^{H}
$$

where $d$ is the Euclidean distance between closest symbols in the particular constellation of $\mathbf{s}$. Thus, the optimization problem in (3) reduces to

$$
\begin{array}{ll}
\underset{\mathbf{W}_{T}}{\operatorname{Maximize}} & \left|\mathbf{I}+\frac{\gamma d^{2}}{4 n_{T}} \mathbf{W}_{T}^{H} \mathbf{R} \mathbf{W}_{T}\right|, \\
\text { subject to } & \left\|\mathbf{w}_{T}[n]\right\|=1, \quad n=1, \ldots, n_{T} .
\end{array}
$$

which resembles the precoder design problem in conventional MIMO systems [11], with the additional constraint in the energy of the columns of $\mathbf{W}_{T}$.

Fortunately, the individual energy constraints can be easily satisfied. In particular, writing the singular value decomposition (SVD)

$$
\mathbf{W}_{T}=\mathbf{U}_{T} \boldsymbol{\Lambda} \mathbf{V}_{T}^{H}
$$

where $\mathbf{U}_{T}, \mathbf{V}_{T}$ are $n_{T} \times n_{T}$ unitary matrices and

$$
\boldsymbol{\Lambda}=\operatorname{diag}\left(\left[\lambda_{1}, \ldots, \lambda_{n_{T}}\right]\right),
$$

contains the singular values, it is easy to see that the determinant in (6) does not depend on the singular vectors $\mathbf{V}_{T}$. Therefore, we can choose $\mathbf{V}_{T}$ as any unitary matrix with constant norm elements, such as the Fourier matrices in (4) or the Walsh-Hadamard matrix, which ensures [12]

$$
\left\|\mathbf{w}_{T}[n]\right\|^{2}=\frac{\operatorname{Tr}\left(\boldsymbol{\Lambda}^{2}\right)}{n_{T}} \quad n=1, \ldots, n_{T} .
$$

With this choice of $\mathbf{V}_{T}$, (6) can be rewritten as

$$
\underset{\mathbf{U}_{T}, \boldsymbol{\Lambda}}{\operatorname{aximize}}\left|\mathbf{I}+\frac{\gamma d^{2}}{4 n_{T}} \boldsymbol{\Lambda}^{2} \mathbf{U}_{T}^{H} \mathbf{R} \mathbf{U}_{T}\right| \quad \text { s.t. } \quad \operatorname{Tr}\left(\boldsymbol{\Lambda}^{2}\right)=n_{T},
$$

\footnotetext{
${ }^{5}$ For instance, it is easy to prove that for low SNR values, these errors dominate the actual error probability.
} 

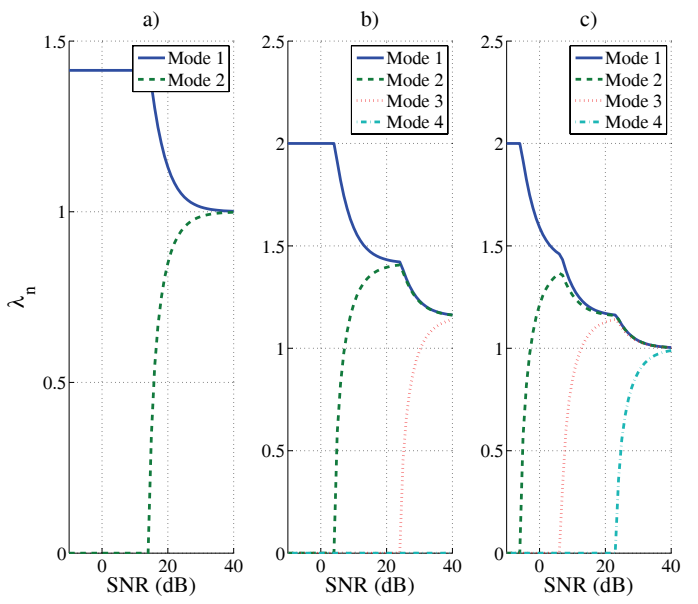

Fig. 4. Spatial power distribution under three different scenarios. a) $n_{T}=2, d_{A}=0.1 \lambda$. b) $n_{T}=4, d_{A}=0.1 \lambda$. c) $n_{T}=4$, $d_{A}=0.25 \lambda$.

and its solution is obtained from standard majorization results [12]. Specifically, writing the eigenvalue (EV) decomposition of $\mathbf{R}$ as

$$
\mathbf{R}=\mathbf{U}_{\mathbf{R}} \boldsymbol{\Sigma}^{2} \mathbf{U}_{\mathbf{R}}^{H}
$$

the optimal beam directions are directly given by $\mathbf{U}_{T}=\mathbf{U}_{\mathbf{R}}$, whereas the optimal power allocation is obtained from a standard water-filling technique [13]

$$
\lambda_{n}^{2}=\left(\kappa-\frac{4 n_{T}}{\gamma d^{2} \sigma_{n}^{2}}\right)_{+}, \quad n=1, \ldots, n_{T},
$$

where $\sigma_{n}^{2}$ are the eigenvalues of $\mathbf{R},(x)_{+}=\max (0, x)$, and $\kappa$ is the water level, which is chosen to satisfy $\operatorname{Tr}\left(\boldsymbol{\Lambda}^{2}\right)=\sum_{n=1}^{n_{T}} \lambda_{n}^{2}=n_{T}$.

Here we must note that, if the number of active modes $(r)$ is lower than $n_{T}$, i.e., if the optimal $\mathbf{W}_{T}$ is rank-deficient, we can obtain similar results by means of only $r$ beamformers. However, this would require the change of the time-precoding matrix G. On the other hand, the rank-deficiency of $\mathbf{W}_{T}$ does not suppose any major problem to transmit $n_{T}$ symbols in $n_{T}$ channel uses.

To summarize, the proposed scheme resembles the statistical waterfilling approach for conventional MIMO systems, i.e., waterfilling over the channel correlation matrix. However, we only transmit a stream of data, and the optimal directions are combined by means of the matrix $\mathbf{V}_{T}$. Finally, it is interesting to point out that, in the case of uncorrelated channels or very high signal to noise ratios $\gamma$, the optimal space encoder is given by any unitary matrix $\mathbf{W}_{T}$. In these cases, the proposed scheme is equivalent to the orthogonal beam division multiplexing (OBDM) technique presented in [2] for the case of i.i.d. (spatially uncorrelated) MIMO Rayleigh channels, which provides full-diversity and maximum coding gain.

\section{SIMULATION RESULTS}

In this section, the performance of the proposed transmission scheme is evaluated by means of simulations. We consider a block-fading model in which the propagation coefficients remain constant for a coherence interval of $L=P n_{T}$ symbols (i.e., the frame duration in Fig. 3). The transmit correlation matrix has been obtained from the

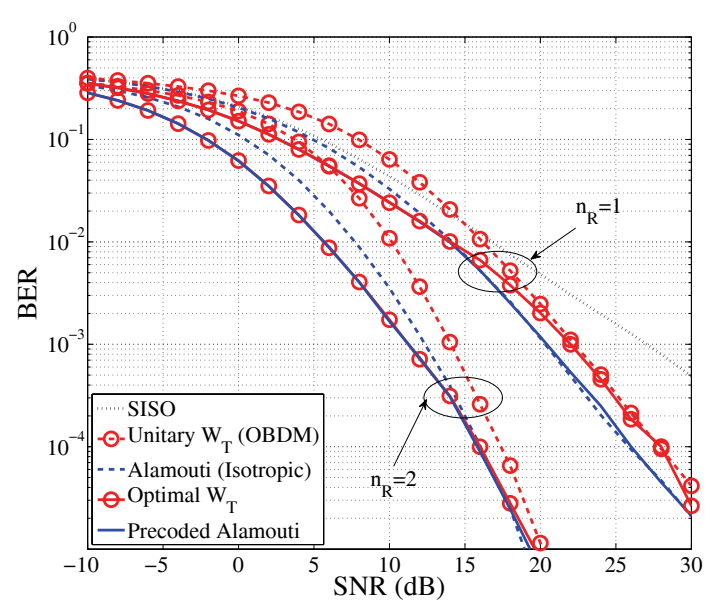

Fig. 5. BER curves for a system with $n_{T}=2$ antennas spaced $d_{A}=0.1 \lambda$ and ML decoding.

Jakes model with antenna spacing of $d_{A}=0.1 \lambda$ or $d_{A}=0.25 \lambda$, where $\lambda$ is the wavelength. In all the examples we have used QPSK signals, and the performance of the proposed scheme has been compared with a SISO system; with the OBDM technique [2], which uses the optimal time encoder $\mathbf{G}$ but does not take into account the spatial correlation (i.e., $\mathbf{W}_{T}$ is unitary); and with conventional MIMO systems using well-known space-time block codes (STBCs), which can be linearly precoded in order to exploit the transmit correlation [11].

The spatial power distribution provided by the water-filling technique under three different scenarios is illustrated in Fig. 4. As can be seen, the transmission scheme varies from pure beamforming for very low SNRs, which means using $n_{T}$ identical beamformers, to isotropic radiation for high SNRs, which is equivalent to the OBDM scheme ( $\mathbf{W}_{T}$ unitary).

In the first example, we have considered a system with $n_{T}=2$ transmit antennas spaced $d_{A}=0.1 \lambda$ and ML decoding. The simulation results are shown in Fig. 5, where we also show the results for the well-known Alamouti scheme [14]. As can be seen, the performance of the proposed scheme is almost identical to that of the Full-MIMO system with Alamouti coding. However, as previously pointed out, the proposed scheme only requires one RF chain at the transmitter and receiver. Finally, as expected, the gain provided by the knowledge of the transmit correlation decreases with the SNR.

In the second example, we have evaluated the performance of the proposed scheme in a $4 \times 4$ MIMO system with antenna spacing at the transmitter side of $d_{A}=0.1 \lambda$. In this case we have employed linear MMSE receivers to show that, although the proposed scheme has been obtained assuming optimal ML receivers, it also provides good results when a suboptimal receiver is used. ${ }^{6}$ The results, which are compared to that of a Full-MIMO system using a quasi-orthogonal STBC (QSTBC) [15] are shown in Fig. 6, where we can observe that the performance of the proposed scheme is almost identical to that of the QSTBC design with optimum linear precoding.

Finally, the impact of the antenna spacing is illustrated in Fig. 7, where the previous experiment has been repeated for $d_{A}=0.25 \lambda$.

\footnotetext{
${ }^{6}$ Of course, the optimal transceiver under the assumption of linear receivers can differ from the solution presented in this paper.
} 


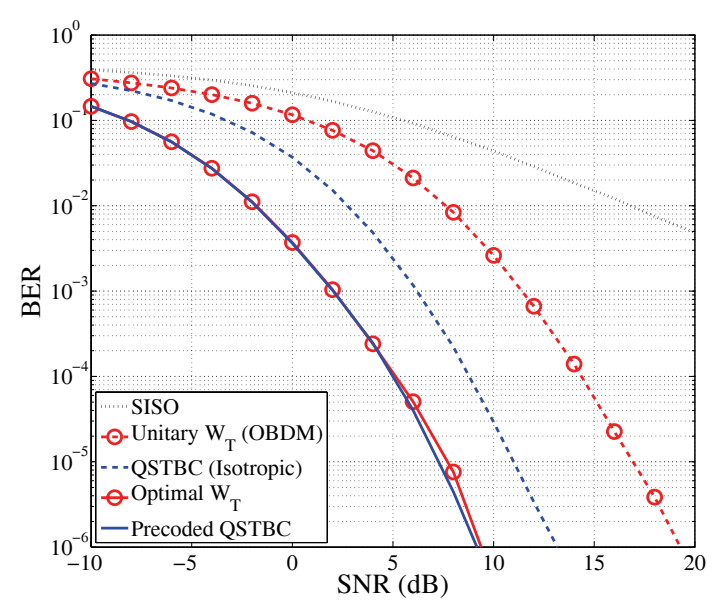

Fig. 6. BER curves for a system with $n_{T}=n_{R}=4$ antennas spaced $d_{A}=0.1 \lambda$ and linear MMSE decoding.

As expected, the gap between the proposed scheme and OBDM decreases with $d_{A}$. However, for $d_{A}=0.25 \lambda$ and practical BER values, the gain provided by the proposed scheme is about $5 \mathrm{~dB}$.

\section{CONCLUSION}

In this paper we have proposed a Tx-Rx scheme for a novel MIMO transceiver that performs signal combining in the radio-frequency domain. The main advantage of this topology is that, after combining in the RF path, there is only one signal stream for baseband processing. Consequently, the power consumption, size and system cost can be significantly reduced compared to a conventional (full baseband) MIMO system. In particular, we have considered the case of perfect channel state information (CSI) at the receiver and statistical CSI at the transmitter. The proposed scheme is based on two different encoders. On the one hand, the time encoder operates at the symbol rate and distributes the information symbols by means of a shifted version of the Fourier matrix. On the other hand, the spatial encoder provides the optimal directions (beamformers or RF weights) for the transmission of the time-precoded symbols. In summary, equipped with the proposed algorithms, the RF adaptive antenna combining architecture is an interesting, low cost, and compact solution for MIMO processing; which provides much better performance than a single antenna system and can even be competitive with full MIMO designs in some realistic scenarios.

\section{REFERENCES}

[1] F. Ellinger, Radio Frequency Integrated Circuits and Technologies, Springer-Verlag, Berlin, 2007.

[2] I. Santamaría, V. Elvira, J. Vía, D. Ramírez, J. Pérez, J. Ibáñez, R. Eickhoff, and F. Ellinger, "Optimal MIMO transmission schemes with adaptive antenna combining in the RF path," in 16th European Signal Processing Conference (EUSIPCO), Lausanne, Switzerland, August 2008.

[3] J. Vía, V. Elvira, I. Santamaría, and R. Eickhoff, "Minimum BER beanforming in the RF domain for OFDM transmissions and linear receivers," in IEEE Int. Conf. on Acoustics, Speech and Sig. Proc., Taipei, Taiwan, Apr. 2009.

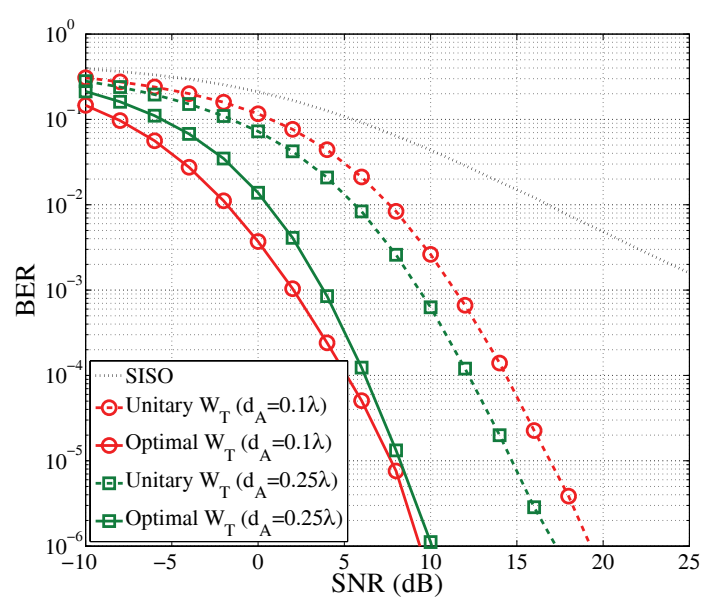

Fig. 7. Effect of the antenna spacing. $n_{T}=n_{R}=4$ and linear MMSE decoding.

[4] J. Vía, V. Elvira, I. Santamaría, and R. Eickhoff, "Analog antenna combining for maximum capacity under ofdm transmissions," in IEEE International Conference on Communications (ICC 2009), Dresden, Germany, June 2009.

[5] X. Ma and G. B. Giannakis, "Complex field coded MIMO systems: performance, rate, and trade-offs," Wireless Comm. and Mobile Computing, vol. 2, no. 7, pp. 693-717, 2002.

[6] Y. Xin, Z. Wang, and G.B. Giannakis, "Space-time diversity systems based on linear constellation precoding," IEEE Trans. on Wireless Comm., vol. 2, no. 2, pp. 294-309, Mar. 2003.

[7] S. Barbarossa, Multiantenna Wireless Communication Systems, Artech House Publishers, 2005.

[8] X. Giraud, E. Boutillon, and J.C. Belfiore, "Algebraic tools to build modulation schemes for fading channels," IEEE Trans. on Inf. Theory, vol. 43, no. 3, pp. 938-952, Mar. 1997.

[9] Vahid Tarokh, Nambi Seshadri, and A. Robert Calderbank, "Space-time codes for high data rate wireless communications: Performance criterion and code construction," IEEE Trans. on Inf. Theory, vol. 44, no. 2, pp. 744-765, Mar. 1998.

[10] G. Jöngren, M. Skoglund, and B. Ottersten, "Combining beamforming and orthogonal space-time block coding," IEEE Trans. on Inf. Theory, vol. 48, no. 3, pp. 611-627, Mar. 2002.

[11] E. Biglieri, R. Calderbank, A. Constantinides, A. Goldsmith, A. Paulraj, and H. Vincent Poor, MIMO Wireless Communications, Cambridge University Press, NY, USA, 2007.

[12] D. P. Palomar and Y. Jiang, "MIMO transceiver design via majorization theory," Found. Trends Commun. Inf. Theory, vol. 3, no. 4, pp. 331-551, 2006.

[13] H. Sampath and A. Paulraj, "Linear precoding for space-time coded systems with known fading correlations," IEEE Communications Letters, vol. 6, no. 6, pp. 239-241, June 2002.

[14] S.M. Alamouti, "A simple transmit diversity technique for wireless communications," IEEE Journal on Selected Areas in Communications, vol. 45, no. 9, pp. 1451-1458, 1998.

[15] H. Jafarkhani, Space-Time Coding: Theory and Practice, Cambridge University Press, 2005. 